\title{
Crystallization process of scorodite at hydrothermal conditions
}

\author{
XIANGYU ZHU, PEI CHANG, SILIANG LI, H. HENRY \\ TENG*
}

Institute of Surface-Earth System Science, Tianjin University, Tianjin 300072, China

(*correspondence: huihenry.teng@tju.edu.cn)

Scorodite $\left(\mathrm{FeAsO}_{4} \cdot 2 \mathrm{H}_{2} \mathrm{O}\right)$ is a common arsenic-fixating mineral in soils, tailings, and sediments and a promising arsenicstorage medium in the metallurgy industry owing to the mineral's low solubility. As such, understanding the mineralization behavior of scorodite is crucial to depict geochemical cycling of arsenic at Earth's surface environments. Here we performed hydrothermal synthesis experiments to accelerate the precipitation rate and probed the process of scorodite crystallization at various chemical conditions using a comprehensive approach combining various experimental techniques including AFM, TEM, XANES, and dynamic nano-particle size analysis. The results showed that the crystallization of scorodite proceeds via precursors of amorphous phases followed by a phase transition to establish long-range structural orders regardless of solution chemistry, instead of through the traditional pathways described by the classic crystallization theory. In addition, the intermediates of the phase transition differ when the valence of the Fe source changes, e.g., solutions with $\mathrm{Fe}^{2+}$ precipitate in the sequence of amorphous $\mathrm{Fe}(\mathrm{II})-\mathrm{Fe}(\mathrm{III})-\mathrm{As}(\mathrm{V})$ phases (still stable after 2 months at 22-23 ${ }^{\circ} \mathrm{C}$ ), spindle aggregates of fibrous scorodite crystals (up to 100 $\mu \mathrm{m}$ ), and the final pseudo-octahedral crystals; while solutions with $\mathrm{Fe}^{3+}$ first precipitate amorphous $\mathrm{Fe}(\mathrm{III})-\mathrm{As}(\mathrm{V})$ phases (partial crystallization occurs after 2 months at $22-23{ }^{\circ} \mathrm{C}$ ), and then the bended dumbbell-shape scorodite aggregates (up to $3 \mu \mathrm{m}$ ). Dynamic particle size analysis revealed that the scorodite nucleus will stop growing at a certain size (usually around tens of nanometer) dependent on temperature and saturation index and can be metastable for up to several days. Amorphous phase starts to transform to crystals following the collapse of this metastable state.

Acknowledgement: This work is financial supported by the National Natural Science Foundation of China (Grant Nos. 41802032, 41830859, and 41861144026) 